\title{
Two-Dimensional Mapping of Chemical Information at Atomic Resolution
}

\author{
M. Bosman, ${ }^{1}$ V. J. Keast, ${ }^{2, *}$ J. L. García-Muñoz, ${ }^{3}$ A. J. D’ Alfonso, ${ }^{4}$ S. D. Findlay, ${ }^{4}$ and L. J. Allen ${ }^{4}$ \\ ${ }^{1}$ Electron Microscope Unit, The University of Sydney, NSW 2006, Australia \\ ${ }^{2}$ School of Mathematical and Physical Sciences, The University of Newcastle, Callaghan, NSW 2308, Australia \\ ${ }^{3}$ Institut de Ciència de Materials de Barcelona, CSIC, Campus Universitari de Bellaterra, E-08193 Bellaterra, Spain \\ ${ }^{4}$ School of Physics, University of Melbourne, Victoria 3010, Australia
}

(Received 8 June 2007; published 22 August 2007)

\begin{abstract}
The simultaneous measurement of structural and chemical information at the atomic scale provides fundamental insights into the connection between form and function in materials science and nanotechnology. We demonstrate structural and chemical mapping in $\mathrm{Bi}_{0.5} \mathrm{Sr}_{0.5} \mathrm{MnO}_{3}$ using an aberrationcorrected scanning transmission electron microscope. Two-dimensional mapping is made possible by an adapted method for fast acquisition of electron energy-loss spectra. The experimental data are supported by simulations, which help to explain the less intuitive features.
\end{abstract}

DOI: 10.1103/PhysRevLett.99.086102

In his famous talk, Plenty of Room at the Bottom (1959) [1], Richard P. Feynman said "It would be very easy to make an analysis of any complicated chemical structure; all one would have to do would be to look at it and see where the atoms are. The only trouble is that the electron microscope is 100 times too poor... I put this out as a challenge: Is there no way to make the electron microscope more powerful?" Feynman was talking about a conventional transmission electron microscope and considerable progress has been made to meet this challenge in the intervening period. We will consider the use of a highresolution scanning transmission electron microscope (STEM) which, rather than illuminating a specimen with a plane wave, scans a small Ångström-sized probe across the sample. At each probe position, electrons which have been scattered through large angles, usually by interaction with a phonon ( $Z$-contrast imaging), can be recorded to obtain a projection of the atomic structure. Electron energy-loss (EEL) spectra can be simultaneously recorded to provide chemical identification and explore local bonding and other electronic properties [2]. Research in nanoscience and technology, protein structure and folding, and the structure of cell membranes, to name just a few, are all areas where knowledge of structure and function at the atomic level is essential.

The correlation of structure, composition, and physical properties using high-resolution STEMs has predominantly been achieved by using the $Z$-contrast image as a probe position reference to record energy-loss spectra at select, structurally significant points [3,4]. With the latest generation of aberration-corrected microscopes [5], recording energy-loss scans along a contiguous sequence of points is possible [6,7], although it is not routine. Overcoming the restriction of EEL studies to predetermined regions of interest will enable a broader investigation of structure-composition-property relations. Implementing this is complicated by recent suggestions that high-resolution STEM images, both Z-contrast and EEL images, do not always admit direct, visual interpretation
PACS numbers: 68.37.Lp, 02.50.Sk, 61.85.+p, 79.20.Uv

[4,8-13]. There are three interrelated reasons for this: (i) the elastic scattering or dynamical spreading of the electron probe; (ii) the electron "absorption" (due to phonon excitation); and (iii) the delocalized and partially coherent nature of the inelastic scattering processes, which can lead to a nonintuitive connection between the probe density in the sample and the size of the recorded signal. These complications are particularly difficult to identify if only point or line scan data are available [14]. EEL mapping in two dimensions, as demonstrated here, allows more convincing comparisons with simulations based on shape than is possible for line scans, and will help our understanding of these processes.

The restriction to EEL line scans at atomic resolution arises because the scattering cross section for innershell ionization processes is relatively low. Adequate counting statistics for Z-contrast images are obtained with millisecond dwell times, whereas dwell times for EEL spectra need to be of the order of tenths of a second, comparable to the time scale on which beam damage, specimen drift, and other mechanical instabilities manifest themselves. The construction of highly stable microscope environments and the ongoing developments in aberration correction (which enable an increase in beam current) are continually improving the situation and, as we report here, by carefully eliminating systematic noise in the EEL spectral acquisition and using multivariate statistical analysis techniques, it is now possible to obtain two-dimensional compositional maps at atomic resolution.

The specimen used for the present study is $\mathrm{Bi}_{0.5} \mathrm{Sr}_{0.5} \mathrm{MnO}_{3}$, a new material that shows colossal magnetoresistant behavior [15]. It is also one of the few materials that shows "charge ordering" at room temperature [16-18]. The compound was synthesized from high-purity precursors by several sintering and pressing cycles as described elsewhere [19]. Neutron and x-ray powder diffraction showed that the material is not an ideal perovskite, but possesses lattice distortions that are most evident in the inclination of the oxygen octahedra about the manganese 
sites. The bismuth and strontium cations are distributed at equivalent lattice sites in a nonordered manner throughout the compound.

The specimen was imaged along the three zone axes $\langle 001\rangle,\langle 110\rangle$ and $\langle 111\rangle$ (where the labeling is based on the approximate cubic perovskite structure). The data were acquired using the VG HB501 dedicated STEM at the SuperSTEM facility in Daresbury, UK. This $100 \mathrm{kV}$ machine, fitted with a Nion spherical aberration corrector and Gatan Enfina EEL detector, has enjoyed much success in structure determination at atomic resolution using $Z$-contrast imaging [20,21]. The probe-forming semiangle and EEL detector collection semiangle were both around 24 mrad. EEL spectra were taken with a $0.2 \mathrm{~s}$ collection time, giving a total acquisition time per data set of around 10 min.

EEL spectrum images from a rectangular specimen area $[22,23]$ were acquired with the method of binned gain averaging. As will be discussed elsewhere, this method improves the speed with which EEL spectra are acquired, while optimally suppressing systematic detector gain. With binned gain averaging, no gain or dark current corrections are used during EEL acquisition; these corrections are done afterwards. The spectra are acquired with full onchip binning, while the position of the EEL spectra on the detector CCD is constantly varied. Postacquisition alignment of the spectra corrects systematic detector gain by averaging correlated intensity in the spectral channels. Thermal excitation of charge in the CCD pixels-dark current-is corrected for by the postacquisition subtraction of a constant value from all spectra. Figure 1 shows a typical example of an EEL spectrum after alignment and dark current subtraction. The spectral features of interest, the oxygen $K$ edge and the manganese $L_{2,3}$ edge, are situated on a sloping background signal that should be

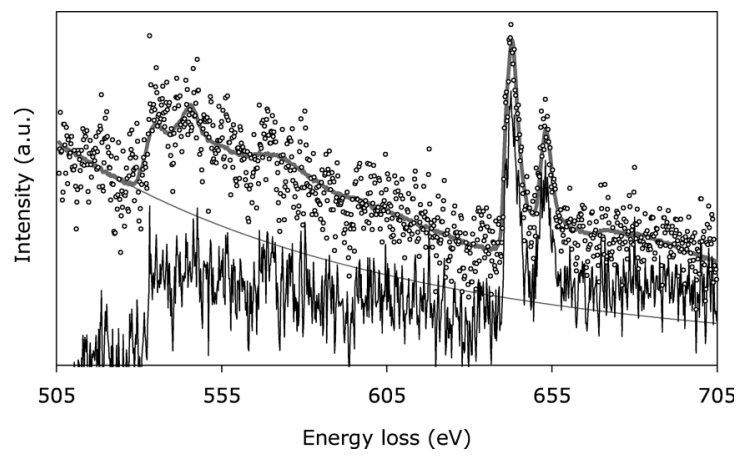

FIG. 1. An example of a single EEL spectrum, taken from the left-hand corner of the EEL data set in Fig. 2. The circles show the raw spectrum after subtracting the dark current. The thick gray line is the principal component analysis reconstruction of the raw spectrum, as calculated from the first three principal components of the whole EEL data set of Fig. 2. The thin gray line is a power-law fit to the reconstructed spectrum, using a $20 \mathrm{eV}$ wide window before the oxygen $K$ edge. The thin black line shows the background-subtracted raw spectrum, which is used for further analysis. removed to obtain the accurate oxygen or manganese intensities. The relatively low signal-to-noise ratio of the individual spectra prevents an accurate fit of a power-law curve, which is the conventional model to remove the background signal. The robustness of the background fit is greatly enhanced by first applying principal component analysis (PCA) to remove the random spectral noise [24]. As shown in Fig. 1, power-law fits are applied to the preedge intensities of the PCA-reconstructed spectra and these background signals are subtracted from the original spectra. This routine is applied to extract both the oxygen signal and the manganese signal. The backgroundsubtracted oxygen and manganese intensity for all spectra were integrated over a $30 \mathrm{eV}$ window above their respective thresholds to produce EEL maps. This energy range avoids major multiple scattering effects which are strongest at higher energy losses. Figures $2-4$ show experimen-
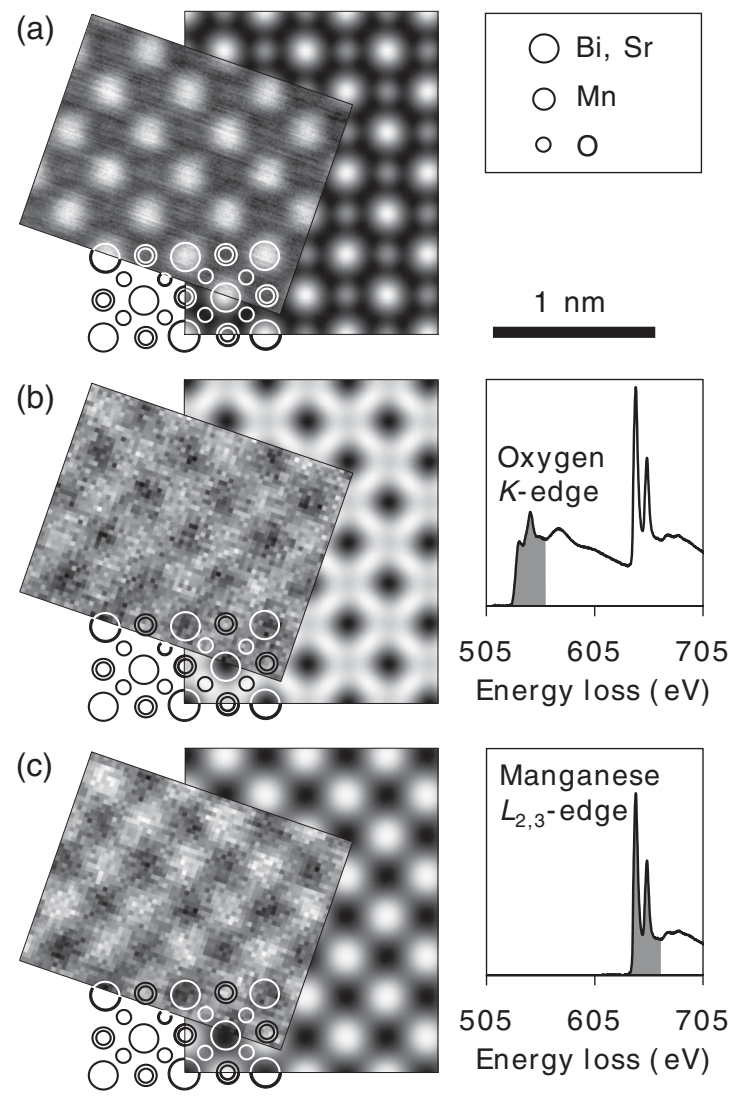

FIG. 2. Comparison between experiments (tilted images) and simulations of $\mathrm{Bi}_{0.5} \mathrm{Sr}_{0.5} \mathrm{MnO}_{3}$ oriented along the $\langle 001\rangle$ zone axis. (a) Z-contrast, (b) oxygen $K$ edge and (c) manganese $L_{2,3}$ edge STEM images. The atomic structure is indicated. The EEL maps were generated by integrating the EEL spectra over a $30 \mathrm{eV}$ window above the respective ionization threshold. This is shown in gray shading on the spectra to the right, which are summations of all the spectra in the adjacent EEL maps. The $Z$-contrast image is the summation of three cross-correlated single images, taken from the same area, with the same experimental settings as the EEL maps. The simulations assume a $330 \AA$ thick sample. 
tal and simulated $Z$-contrast images, together with EEL maps of the oxygen and manganese signal for the three orientations. The acquisition parameters were identical for the $Z$-contrast and EEL measurements. The specimen thicknesses, determined using low-loss spectra from the same area, are estimated at $31.3-35.8 \mathrm{~nm}, 12.0-12.5 \mathrm{~nm}$, and $35-45 \mathrm{~nm}$ for the $\langle 001\rangle,\langle 110\rangle$, and $\langle 111\rangle$ oriented samples respectively - the large spread in the last being attributed to beam damage as will be discussed later.

The "orth 1" atomic structure at $300 \mathrm{~K}$ given in Ref. [19] is used as input to the direct simulations. These are Bloch wave simulations based on effective inelastic scattering potentials calculated using the method described in Ref. [25], accounting for the $\mathrm{Bi} / \mathrm{Sr}$ columns using fractional occupancy. The aberration-balanced system is modeled as aberration-free within the $24 \mathrm{mrad}$ probe-forming aperture - an assumption which may account for some of the discrepancy between measured and simulated resolutions. The $Z$-contrast images were simulated for a $60-$ $160 \mathrm{mrad}$ annular dark field detector. The EEL images were simulated with a 24 mrad detector semiangle and a $30 \mathrm{eV}$ collection window above the ionization threshold. For the present experiment, the demagnification of the effective electron source was slightly lower than that for optimal resolution to enhance the current in the probe for better EEL counting statistics. To account for this finite width of the effective source, all simulated images were convolved with a Gaussian of half-width $0.8 \AA$ [26]. This number, obtained by best visual comparison with the experimental data, still permits the imaging of the $0.14 \mathrm{~nm}$ dumbbell structure in silicon along the $\langle 110\rangle$ zone axis, consistent with results obtained from this STEM under similar operating conditions.

A few features of these images warrant comment. In the simulated oxygen image in Fig. 2(b), the signal on the $\mathrm{Mn} / \mathrm{O}$ column is smaller than that on the pure oxygen columns, despite the identical oxygen densities. This is a consequence of the different scattering and absorption caused by the presence of manganese. However, the difference is too small to be evident in the experimental image. More pronounced dechanneling effects can be seen in the oxygen map of Fig. 3(b), where the signal is smallest on the $\mathrm{Bi} / \mathrm{Sr} / \mathrm{O}$ column, though admittedly in this orientation the oxygen density on these columns is half that on the clearly visible pure oxygen columns. More intriguingly in this image, the simulations show evidence of the inclination of the $\mathrm{MnO}_{6}$ octahedra through the alternating displacements in oxygen position when looking along horizontal rows in the figure. A hint of this behavior is also seen in the experimental data.

Specimen damage was only observed in the $\langle 111\rangle$ orientation. The experimental oxygen map in Fig. 4(b) has less intensity in the lower part, towards the end of the EEL acquisition, indicating a decrease of oxygen content. The manganese map is more uniform. However, a shoulder on the low-energy side of the manganese $L_{3}$ edge becomes more pronounced for spectra in the lower part of the data

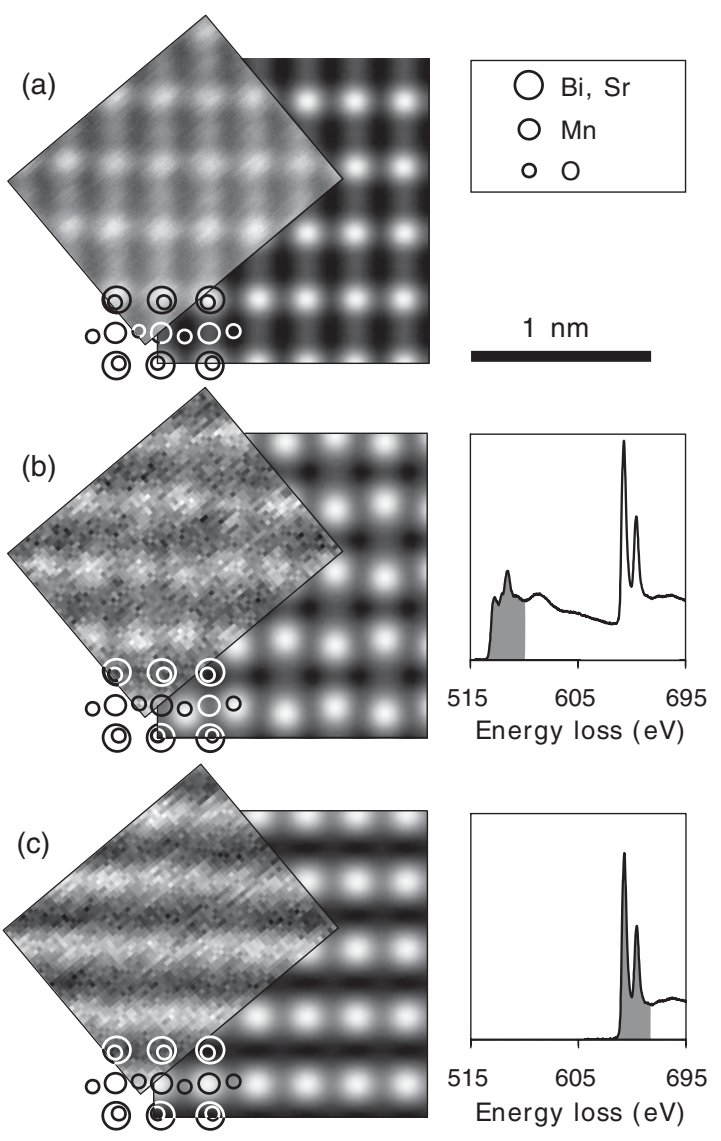

FIG. 3. As Fig. 2 but with the $\mathrm{Bi}_{0.5} \mathrm{Sr}_{0.5} \mathrm{MnO}_{3}$ specimen oriented along the $\langle 110\rangle$ zone axis. (a) Z-contrast, (b) oxygen $K$ edge and (c) manganese $L_{2,3}$ edge STEM images. The zigzag pattern of the oxygen columns is most noticeable in this specimen orientation and is caused by the tilted $\mathrm{MnO}_{6}$ octahedra. The simulations assume a $120 \AA$ thick sample.

set. Systematic studies $[27,28]$ in combination with the oxygen map indicate the partial reduction of the manganese cations due to electron beam-induced specimen damage. This effect necessitated the selection of a relatively thick area for the $\langle 111\rangle$ measurements of Fig. 4. The fact that no beam damage was observed in the $\langle 001\rangle$ and $\langle 110\rangle$ orientation indicates that the threshold energy for specimen damage is orientation dependent, which can be explained from the highly anisotropic structure of the material. Of all three Figs. 2-4, the $\langle 111\rangle$ orientation with its $35-45 \mathrm{~nm}$ thickness gives the least intuitive EEL maps. The oxygen image, Fig. 4(b), has high intensity on the oxygen columns, as might be expected. Propagation effects are of secondary significance to this image; a similar spatial distribution is observed in simulations for a single cell thickness. In Fig. 4(c), however, the manganese signal is smallest on the $\mathrm{Bi} / \mathrm{Sr} / \mathrm{Mn}$ columns, the only columns containing manganese. The gray scale images, plotting minimum to maximum as black to white, obscure the relatively low contrast variation which is more obvious in the experimental than the simulated data. The dip at the $\mathrm{Bi} / \mathrm{Sr} / \mathrm{Mn}$ columns is a consequence of the strong absorption of that column; the 


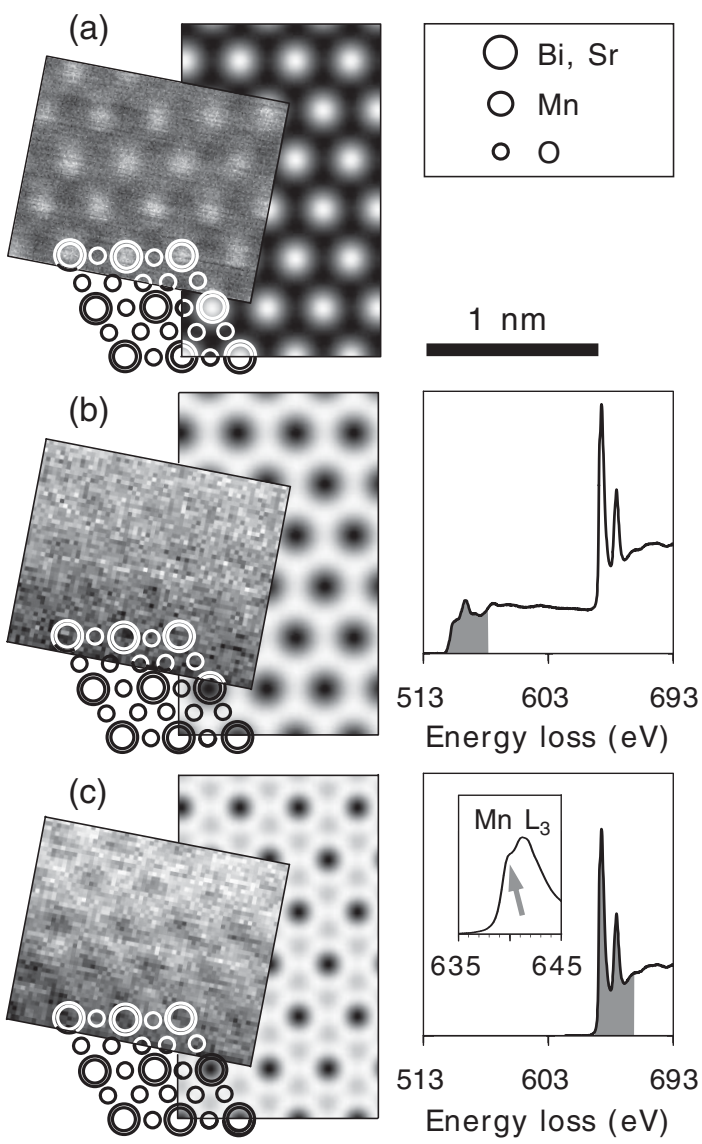

FIG. 4. Same as Figs. 2 and 3 but with the $\mathrm{Bi}_{0.5} \mathrm{Sr}_{0.5} \mathrm{MnO}_{3}$ specimen oriented along the $\langle 111\rangle$ zone axis. (a) Z-contrast, (b) oxygen $K$ edge and (c) manganese $L_{2,3}$ edge STEM images. The shoulder on the low-energy side of the manganese $L_{3}$ edge (inset) becomes more pronounced from top to bottom in the EEL data, indicating a reduction of the manganese cations during acquisition. The simulations assume a $400 \AA$ thick sample.

strong signal between these columns is a consequence of probe spreading towards the manganese-containing columns. The brighter ring about the $\mathrm{Bi} / \mathrm{Sr} / \mathrm{Mn}$ columns indicates the range of significant interaction with the delocalized ionization potential.

In summary, we have shown that the ability to record two-dimensional EEL maps greatly aids the interpretation of compositional information in terms of sample and chemical structure. Aberration-corrected STEMs are now in a position to investigate and correlate structural, chemi$\mathrm{cal}$, and functional information at atomic resolution in two dimensions.

V. J. Keast and L. J. Allen acknowledge support by the Australian Research Council. Financial support by the Spanish MEC (No. MAT2006-11080-C02-02) and the FAME Network are also acknowledged. A.L. Bleloch and M.H. Gass are kindly thanked for their assistance at the Daresbury SuperSTEM and M. Watanabe for providing the PCA code. *vicki.keast@newcastle.edu.au

[1] URL http://www.zyvex.com/nanotech/feynman.html.

[2] J. C. H. Spence, Rep. Prog. Phys. 69, 725 (2006).

[3] D. A. Muller, T. Sorsch, S. Moccio, F. H. Baumann, K. Evans-Lutterodt, and G. Timp, Nature (London) 399, 758 (1999).

[4] M. Varela, S. D. Findlay, A. R. Lupini, H. M. Christen, A. Y. Borisevich, N. Dellby, O. L. Krivanek, P. D. Nellist, M. P. Oxley, and L. J. Allen et al., Phys. Rev. Lett. 92, 095502 (2004).

[5] P.E. Batson, N. Dellby, and O. L. Krivanek, Nature (London) 418, 617 (2002).

[6] L. J. Allen, S. D. Findlay, A. R. Lupini, M. P. Oxley, and S. J. Pennycook, Phys. Rev. Lett. 91, 105503 (2003).

[7] A. Bleloch, U. Falke, and M. Falke, Microsc. Microanal. 9, Suppl. 3, 40 (2003).

[8] T. Yamazaki, M. Kawasaki, K. Watanabe, I. Hashimoto, and M. Shiojiri, J. Electron Microsc. 50, 517 (2001).

[9] K. Watanabe, T. Yamazaki, I. Hashimoto, and M. Shiojiri, Phys. Rev. B 64, 115432 (2001).

[10] K. Ishizuka, J. Electron Microsc. 50, 291 (2001).

[11] C. Dwyer and J. Etheridge, Ultramicroscopy 96, 343 (2003).

[12] S.D. Findlay, M. P. Oxley, S. J. Pennycook, and L. J. Allen, Ultramicroscopy 104, 126 (2005).

[13] M. P. Oxley, E. C. Cosgriff, and L. J. Allen, Phys. Rev. Lett. 94, 203906 (2005).

[14] M. P. Oxley, M. Varela, T. J. Pennycook, K. van Benthem, S. D. Findlay, A. J. D'Alfonso, L. J. Allen, and S. J. Pennycook, Phys. Rev. B (to be published).

[15] G. Van Tendeloo, O. I. Lebedev, M. Hervieu, and B. Raveau, Rep. Prog. Phys. 67, 1315 (2004).

[16] K. Dörr, J. Phys. D 39, R125 (2006).

[17] J. L. García-Muñoz, C. Frontera, M. A. García-Aranda, A. Llobet, and C. Ritter, Phys. Rev. B 63, 064415 (2001).

[18] C. Frontera, J. L. García-Muñoz, A. Llobet, M. A. GarcíaAranda, and C. Ritter, J. Phys. Condens. Matter 13, 1071 (2001).

[19] C. Frontera, J. L. García-Muñoz, M. A. García-Aranda, C. Ritter, A. Llobet, M. Respaud, and J. Vanacken, Phys. Rev. B 64, 054401 (2001).

[20] U. Falke, A. Bleloch, and M. Falke, Phys. Rev. Lett. 92, 116103 (2004).

[21] I. Arslan, A. Bleloch, E. A. Stach, and N. D. Browning, Phys. Rev. Lett. 94, 025504 (2005).

[22] C. Jeanguillaume and C. Colliex, Ultramicroscopy 28, 252 (1989).

[23] J.A. Hunt and D. B. Williams, Ultramicroscopy 38, 47 (1991).

[24] M. Bosman, M. Watanabe, D. T. L. Alexander, and V. J. Keast, Ultramicroscopy 106, 1024 (2006).

[25] L. J. Allen, S. D. Findlay, M. P. Oxley, and C. J. Rossouw, Ultramicroscopy 96, 47 (2003).

[26] P. D. Nellist and J. M. Rodenburg, Ultramicroscopy 54, 61 (1994).

[27] L. A. J. Garvie and A. J. Craven, Phys. Chem. Miner. 21, 191 (1994).

[28] J. Verbeeck, O. I. Lebedev, G. V. Tendeloo, and B. Mercey, Phys. Rev. B 66, 184426 (2002). 